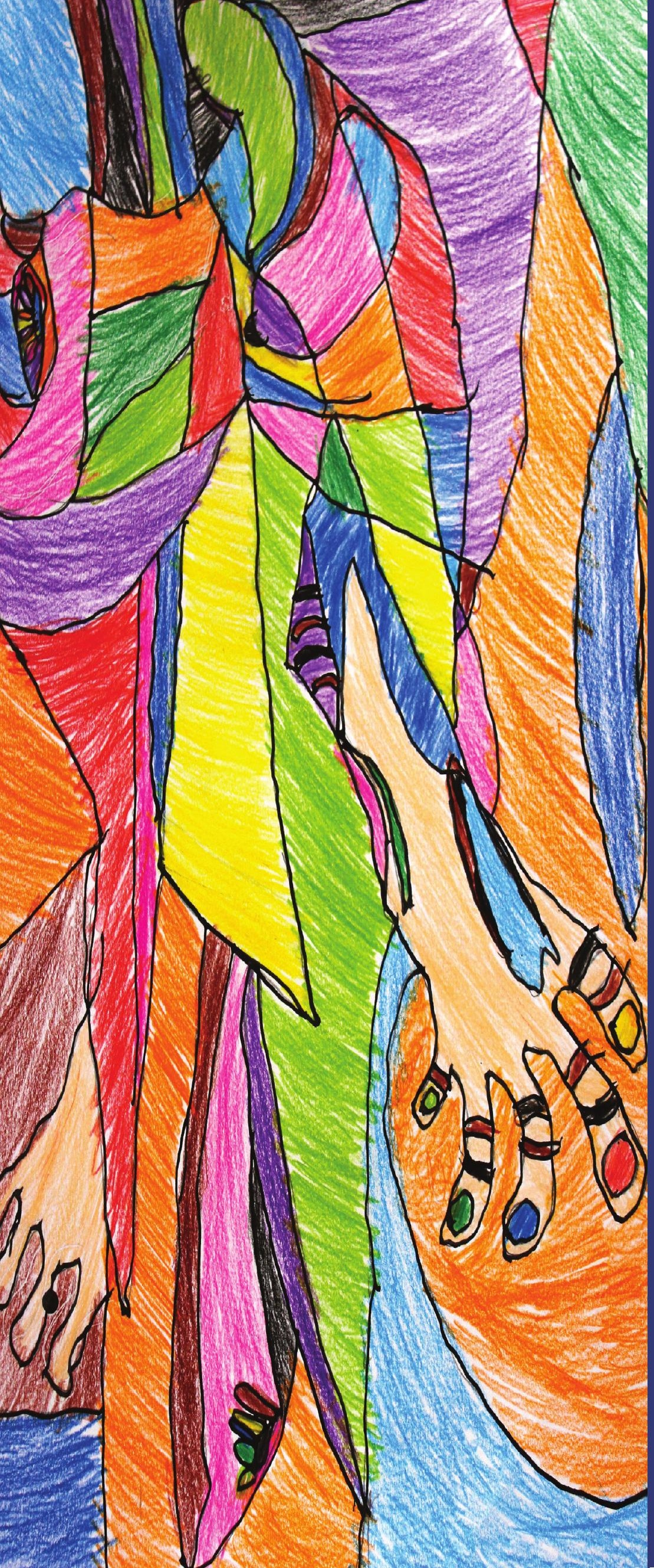

Iván Darío Pinzón Ríos

Fisioterapeuta.

Especialista en Pedagogía Universitaria.

M Sc Ciencias de la Actividad Física y Deporte

Docente Escuela de Fisioterapia Universidad Industrial de Santander.

Carrera 32 N$^{\mathrm{o}}$ 29-31 Facultad de Salud, UIS.

Bucaramanga, Colombia

ivandpr@hotmail.com

Isabel Cristina Tenorio

Arte sin Fronteras - Programa Talentos Especiales 


\section{ACTUALIZACION EN FIBROMIALGIA: IMPLICACIONES NEUROFISIOLÓGICAS Y BIOMECÁNICAS ÚTILES PARA EL ABORDAJE FISIOTERAPÉUTICO}

\section{Update in fibromyalgia: neurophysiological and biomechanical implications useful for a physiotherapy aproach}

\section{RESUMEN}

La Fibromialgia es una patología reconocida como una condición común en la consulta de Fisioterapia y una causa importante de morbilidad a nivel mundial, que cada día va en aumento. Por ello es importante conocer las causas neurofisiológicas y biomecánicas relacionadas con esta enfermedad, para ser tenidas en cuenta en el tratamiento fisioterapéutico, siendo éste el objetivo del presente artículo de revisión. De su profundo análisis y comprensión se deriva una adecuada elección de las modalidades de tratamiento pertinentes para su manejo encaminado a disminuir el impacto de las limitaciones en las actividades funcionales o restricciones en los roles de estos pacientes.

PALABRAS CLAVE

Fibromialgia, Terapia física, Tratamiento, Dolor.

\section{ABSTRACT}

Fibromyalgia is a condition recognized as common in physiotherapy and a major cause of morbidity worldwide, every day it is increasing. It is therefore important to understand the neurophysiological and biomechanical causes related to this disease to be taken into account in the physiotherapy treatment, this being the objective of this review article. From a deep analysis and understanding we can derive a suitable choice of appropriate treatment methods for management of fibromyalgia, aimed at reducing the impact of limitations in activities and restrictions in the roles of these patients. 
a Fibromialgia (FM) es una patología prevalente (Alegre et al., 2010; Arias, 2008), caracterizada de dolor generalizado, fatiga, alteraciones del sueño; algunas condiciones asociadas como migraña, colon irritable, dismenorrea, urgencia urinaria, depresión, falta de concentración causados por factores como la ansiedad, estrés, ejercicio inadecuado, cambios hormonales, alteraciones posturales, fumar, cambios climáticos y traumas físicos y/o emocionales entre otros (Jahan, Nanji, Qidwai y Qasim, 2012; Smith, Harris y Clauw, 2011; Culpepper, 2010; Wolfe, et al, 2010; Perrot, 2008; Contreras y Tamayo, 2005). La encuesta de FM en las Américas mostró que los latinoamericanos deben pasar por más de cinco médicos y esperar más de tres años para obtener el diagnóstico certero; de la población encuestada el 85\% experimentó dolor diseminado una vez a la semana, el $78 \%$ calificó su dolor con puntaje superior a $7 / 10$, el $52 \%$ sintieron limitación en su vida profesional, el 54\% señaló que les impidió trabajar más de diez veces al año, el 34\% admitió sentirse incapaz de trabajar y obtener ingresos, el 30\% perdió su trabajo, el $14 \%$ de los médicos generales reconoció saber poco o nada sobre la patología y el $75 \%$ de los pacientes admitió que no había oído hablar de ésta antes de ser diagnosticado (Hurtado, 2012).

Esta patología es más común en mujeres (Aparicio, et al., 2012) y en Colombia la quinta Encuesta Nacional de Dolor concluyó que la proporción mujer:hombre para FM es de aproximadamente 4:1 (Asociación Colombiana para el Estudio del Dolor, 2010). Según reportes del primer Congreso de Dolor y Anestesia regional del Oriente Colombiano del 2012, la FM ocupó el sexto lugar en las enfermedades dolorosas más comunes (Esteban, 2012). Esta patología es más frecuente en la región andina oriental mientras mayor sea la edad del paciente, siendo usual en mujeres de la raza negra (Hernández y Moreno, 2008). Con relación a la ubicación geográfica, fue mayor la prevalencia en clima templado comparada con los climas cálido y frio; presentando también valores de actividad física menores en esta población (Asociación Colombiana para el Estudio del Dolor, 2010). Por su parte Hurtado citando a Gómez en 2012, estima que cerca al 90\% de las colombianas con FM son subdiagnosticadas y recibe una atención insuficiente (Hurtado, 2012).

Por todo esto, los tratamientos para la FM son diversos y muestran resultados diferentes, unos más aconsejados (mayor evidencia científica) que otros. Con evidencia fuerte están antidepresivos tricíclicos y ciclobenzaprina, ejercicio aeróbico (Schiltenwolf, et al., 2008) mas psicoterapia y tratamientos psicológicos (Chong y $\mathrm{Ng}$, 2009). Con evidencia moderada Analgésicos, Inhibidor selectivo de la recaptación de serotonina (ISRS) y ejercicio aeróbico (Chong y $\mathrm{Ng}$, 2009); con evidencia limitada los inhibidores duales de la recaptación de serotonina y noradrenalina (IDRSN), tropisetrón, ritanserina, 5-hidroxitriptófano, pregabalina, oxibato sódico, hormona del crecimiento, campos electromagnéticos, homeopatía, suplementos dietéticos, balneoterapia y spa (Schiltenwolf, et al., 2008); faltos de evidencia los anti-inflamatorios no esteroideos (AINES), opioides mayores, benzodiacepinas, S-adenosil metionina, corticoides, melatonina, deshidroepiandrosterona, quiropraxia, osteopatía, ozonoterapia (Rivera, Alegre, Nishishinya y Pereda, 2006), hipnosis (Castel, Cascón, Padrol y Rull, 2012; Derbyshire, Whalley y Oakley, 2009)
Gran, 2002).

Por ello el fisioterapeuta debe comprender y evaluar los complejos mecanismos que intervienen en la generación, modulación, amplificación y perpetuación del dolor como base de una intervención terapéutica integral (Moretti, Ezequiel, Marvulle, y Riera, 2011). En este sentido, la Fisioterapia tiene un papel significativo en el tratamiento de esta enfermedad, pues emplea modalidades de tratamiento como el masaje (Castro-Sánchez, et al., 2011a; Castro-Sánchez, et al., 2011b; Castro-Sánchez, et al., 2011c; Kalichman, 2010), electroterapia (Carbonario, Matsutani, Yuan y Marques, 2013), termoterapia (Gauffin, et al., 2013), educación (Ang, et al., 2011; Cedraschi, et al., 2004), ejercicio (Altan, Korkmaz, Bingol y Gunay, 2009; Busch, et al., 2011; Busch, Barber, Overend, Peloso y Schachter, 2007; Cardenas y Ruiz, 2014; Dupree, Adams, Winters-Stone y Burckhardt, 2006; Häkkinen, Häkkinen, Hannonen y Alen, 2001; Jones, et al., 2008; Jones, Burckhardt, Clark, Bennett y Potempa, 2002; Karper, y Hampton, 2006; Kayo, Peccin, Sanches y Trevisani, 2012) balneoterapia e hidroterapia (Evcik, Kizilay y Gokcen, 2002), las cuales en muchos casos son alternativas óptimas para su tratamiento.

Por eso este profesional debe basar su actuar en la comprensión y manejo del movimiento corporal humano ( $\mathrm{MCH}$ ), como elemento esencial de la salud y el bienestar del hombre, optimizando o potencializando el movimiento así como prevenir y recuperar las alteraciones y habilitar/rehabilitar de manera integral a los individuos con el fin de contribuir a la mayor funcionalidad, según lo contempla la Ley 528 de 1999. Las directrices basadas en la evidencia para el manejar FM por parte del terapeuta, se basan en estudios que analizan los componentes del tratamiento de la terapia física que debe incluir la educación, el ejercicio y los medios físicos (Nijs, Mannerkorpi, Descheemaeker y Van Houdenhove, 2010). Este artículo de revisión busca conocer las causas neurofisiológicas y biomecánicas relacionadas con la FM, sirviendo como base para mejorar la comprensión y el tratamiento brindado por el fisioterapeuta en esta patología.

\section{MÉTODO}

Se realizó una revisión de literatura acerca de las causas neurofisiológicas y biomecánicas de la FM, así como la importancia de la fisioterapia en el manejo de dicha patología. La búsqueda se realizó combinando los términos MESH (Medical Subject Headings) Fibromyalgia, pain y physical therapy. Para la revisión de la patología se utilizó la siguiente combinación de descriptores: (fibromyalgia[Title) Abstract]) AND pain[Title/Abstract] teniendo en cuenta que la información se encontrara entre los años $2000-2013$. Para ser incluidos debían ser artículos originales que estudiaran las fisiopatogenia de la enfermedad. Para la revisión de la importancia de la Fisioterapia en el manejo de esta enfermedad, se usó la siguiente combinación de descriptores: (fibromyalgia [Title/Abstract]) AND pain [Title/ Abstract] AND physical therapy [Title/Abstract], sin restricciones de búsqueda. Se seleccionaron y revisaron publicaciones científicas en inglés y español de las bases PUBMED, ELSEVIER y EBSCO que indexan alta producción global en información científica en Salud. Finalmente se combinaron por parejas con el operador OR y se omitieron duplicados de los mismos. La búsqueda también in- 
cluyó fuentes electrónicas, impresas y libros que complementaron la información recopilada; sin embargo algunos de estos últimos tenían un tiempo de publicación anterior al inicialmente establecido, debido a que existen publicaciones relevantes desde los años 90. Del total de publicaciones elegibles [PUBMED (857), ELSEVIER (1) y EBSCO (65)] tras lectura de los resúmenes y títulos, se tomaron aquellos que cumplían con los requisitos para la selección (254); los demás se descartaron por no precisar validez de acuerdo a los criterios establecidos. Después de esto aplicaron 63 referencias según las especificaciones requeridas.

\section{RESULTADOS}

Según la literatura revisada, luego de su lectura y análisis se pudieron establecer los siguientes apartados: breve reseña histórica, causas neurofisiológicas del dolor, causas biomecánicas del dolor, principales alteraciones, sintomatología e importancia de la Fisioterapia en la FM.

\section{Breve Reseña Histórica de la FM}

Esta patología poco conocida ya se referenciaba como la presencia de un conglomerado sintomático en los textos bíblicos muy antiguos (Villagrán, Páez, Campo, Pérez y Salaberri, 200o). Hace unos 150 años, científicos alemanes denominaron "reumatismo muscular" a síntomas asociados a dolores osteomusculares generalizados y la existencia de nódulos dolorosos a la presión. Balfour en 1824 y Valleis en 1841 fueron pioneros en relacionar los puntos dolorosos y el reumatismo; Frorier en 1843 comentó que los pacientes con "reumatismo" tenían puntos musculares "duros" dolorosos a la presión. A finales del siglo XIX, Beard describió un cuadro de dolor generalizado que denominó "miolastenia" y que posteriormente fue considerado como un proceso cercano a la neurastenia (Wolf, 2009). A principios del siglo XX Gowers introdujo el término de "fibromiositis" para describir un cuadro de hipersensibilidad muscular caracterizada por un conjunto de nódulos fibrosos constituidos por tejido colágeno y terminaciones nerviosas extraordinariamente dolorosas a la presión y al esfuerzo mecánico muscular, con etiología diversa. Posteriormente Moldofsky en 1965 comprobó que en los pacientes con "fibromiositis" existía una contaminación de las fases IV del sueño por ondas alfa ampliando el cuadro clínico. En 1972 Smythe organizó los "puntos dolorosos a la presión" o "tender points" y estableció los primeros criterios diagnósticos (Rubio y Paredes, 2004).

La palabra Fibromialgia (FM) fue acuñada en 1976 y proviene de los términos latinos fibro (fibroso), mio (músculo) algia (dolor) (Chaitow, 1995).En los años ochenta se realizan los primeros estudios para definir esta enfermedad y se publican las bases de ésta bajo términos como "Fibromialgia", "síndrome miofascial" y "tendomiopatía generalizada” (Wolf, 2009). En 1987, la Asociación Médica Americana la reconoció como una patología real y una posible causa de la discapacidad (Lee, 2006) la cual ha aumentado significativamente en los últimos años (Caballero, 2010). Otras organizaciones como el National Institutes of Health (NIH) y la Organización Mundial de la Salud (OMS), la han aceptado como entidad clínica legítima, definida según el American College of Reumatology como dolor generalizado con duración mayor o igual a tres meses en al menos 11 de los 18 puntos específicos hipersensibles en el examen (Hernández y Moreno, 2008; Wolfe, et al., 1990) aplicando aproximadamente $4 \mathrm{~kg} / \mathrm{cm} 2$ de presión necesaria para detectar diferencias entre la activación cerebral del dolor, de manera bilateral de los hemicuerpos del paciente (Imamura, Cassius y Fregni, 2009).

Aunque la combinación de estos dos criterios proporciona una sensibilidad del $88 \%$ y una especificidad del $81 \%$ en el diagnóstico de FM, se debe diferenciar de otras causas de dolor musculoesquelético crónico y síntomas adicionales (Wolfe, et al., 1990). Hoy en día también es aceptable una clasificación para la FM, teniendo en cuenta el Índice de Dolor Generalizado (WPI) con puntuación $>7$ y la Escala de la Severidad de los Síntomas (SSS) con puntuación $>5$ o también WPI 3-6 y SSS $>9$ (Wolfe, et al., 2010). En el siglo XXI, se demostró que existe una predisposición genética a desarrollarla mostrando en los estudios cierta agregación familiar (Light, White, Tadler, Iacob y Light, 2012). Según este modelo genético, se supone que en el individuo predispuesto, podría actuar un agente disparador, como un traumatismo (habitualmente a nivel del cuello), o una infección para desencadenarla. Finalmente, los últimos estudios mencionan las alteraciones en la bioquímica del músculo, que puede ser de la contracción muscular o de la transmisión nerviosa a nivel del sistema musculo esquelético, siendo estas muy aceptadas en la actualidad.

\section{Causas Neurofisiológicas del dolor en la FM}

La principal etiología considerada actualmente siguen siendo los cambios en los neurotransmisores tales como la serotonina, la sustancia $\mathrm{P}$, la hormona del crecimiento y el cortisol así como las implicaciones de ésto sobre la regulación autonómica y endocrina (Chaves, 2013; Arias, 2008). La FM causa una sensibilización central, alterando las vías inhibitorias del dolor y los neurotransmisores, reduciendo el umbral de dolor y amplificación de señales sensoriales álgidas (Jahan, Nanji, Qidwai y Qasim, 2012). Por ello inicialmente conviene entender la descripción neuromorfológica acerca del procesamiento de un estímulo doloroso.

Un nocireceptor es una terminación nerviosa con un extremo conectado al sistema nervioso central (SNC) mediante una vaina de mielina (fibra tipo III) o no mielinizada (fibra tipo IV) y el otro recibe aferencias. Según Rubio, las entradas de información se activan de dos formas: 1) Al recibir estímulos mecánicos el receptor es capaz de diferenciarlos de forma que no se activa con los movimientos fisiológicos, presiones locales suaves o estiramientos que no provoquen daño orgánico. 2) Las terminaciones nociceptivas de los músculos y otros tejidos blandos tienen quimiorreceptores y se logra una activación química del nociceptor a sustancias como la bradikinina (BKN), la 5-hidroxitriptamina (5-HT serotonina) y el potasio (K) a concentraciones elevadas. El nociceptor típico responde tanto a estímulos de presión local como la interacción química, sin embargo algunos son activados por un único tipo de estimulación nociceptiva (mecánica o química).

Estos estímulos hacen que existan diferentes tipos de nociceptores, algunos particularmente sensibles a contracciones isquémicas, pues se comprobó que no se desencadenaba estímulo doloroso mediante contracciones fisiológicas (inducidas mediante corrientes), 
2 pero que sí se activaba si la contracción era inducida por oclusión de la arteria que irriga el músculo. Esto sugiere que existe un tipo de nociceptor sensible a la contracción isquémica el cual bien podría ser mediador del dolor en las ciertas estructuras puesto que la disfunción miofascial se manifiesta con una restricción que compromete el aporte vascular local. Por ello cuando existe una disfunción miofascial se compromete también la eliminación de toxinas de forma que siempre está presente un cierto grado de inflamación y se suceden fenómenos de activación de las terminaciones nerviosas a través de receptores B1 lo cual explica el estado hiperálgico. De todos estos receptores, son dos los más implicados, los llamados receptores purinérgicos como el receptor $\mathrm{P}_{2} \mathrm{X}_{3}$ y el receptor vaniloide (VR-1) (Rubio y Paredes, 2004).

Los receptores purinérgicos se unen a moléculas de ATP y de esta forma excitan al nociceptor provocando el estímulo doloroso. Cada vez que una célula del tejido conectivo es lesionada libera ATP y éste a elevadas concentraciones es el responsable del estímulo doloroso. Por tanto, el ATP puede ser considerado como una sustancia indicadora de daño en los tejidos, puesto que los receptores purinérgicos son activados cuando se produce un daño tisular y la necrosis celular subsiguiente está asociada a una liberación de ATP en el medio. Por su parte, el receptor "vaniloide" responde a incrementos de concentración de ión $\mathrm{H}+\mathrm{y}$ al calor. La sensibilidad de estos a los protones desencadena el estímulo doloroso en condiciones en las que el pH local descienda (isquemia o inflamación), común en el foco de restricción miofascial (los denominados "tender points" o puntos gatillo) que lleva implícito un compromiso vascular local. A este cuadro de factores álgidos se añade la fibrosis del tejido conectivo a expensas de la matriz extracelular con reducción del material celular. Los mastocitos o "células cebadas", encargadas de la secreción de serotonina entre otras sustancias, sufren una reducción que implica una disminución de los niveles de serotonina (comprobado en estos pacientes), que contribuye al estado hiperálgico del paciente (Rubio y Paredes, 2004).

El dolor se atribuye a la estimulación de terminaciones nerviosas libres tipo III o tipo IV debida a la acción de diversos neurotransmisores (especialmente sustancia P) (Millea y Holloway, 200o), llegando a la de hiperexcitabilidad de las astas posteriores medulares, la cual provoca aumento en la transmisión de aferencias nociceptivas a través de las vías ascendentes espinotalámicos lateral y anterior. Luego la segunda neurona hace sinápsis en el núcleo ventral posterolateral del tálamo con una tercera neurona cuyo axón se dirige hasta alcanzar el área somatoestésica o de la sensibilidad general (también conocida como área 1, 2 y 3) donde finalmente se percibe de manera consiente la sensación dolorosa (Sluka, 2012).

Posteriormente es enviada al área de asociación Psicosomatoestésica (también denominada área sensitiva secundaria o área 5 y7) y al área motora de la corteza cerebral poniendo en marcha mecanismos antiálgicos que perpetúan la hipomovilidad de los tejidos desde los que se inició el impulso doloroso así como una hipertonía muscular a nivel paravertebral del segmento medular responsable del estímulo y como respuesta, hay un círculo vicioso que perpetúa la lesión en el tejido fascial. Cuando este mecanismo es crónico, se activan las vías ascendentes del dolor incluso frente a estímulos no nociceptivos (alodinia) e incluso percibe como "muy intensos" dolores que en condiciones normales apenas serían perceptibles (hiperalgesia) y a nivel químico, esta sensibilización central se traduce en un aumento de sustancia P en el líquido cefalorraquídeo (Sluka, 2012; Tovar, 2005).

También se ha confirmado las anomalías en el flujo sanguíneo gracias a la tomografía computarizada por emisión de fotón único (SPECT) donde han iluminado las partes cerebrales tradicionalmente ligadas al dolor en pacientes con FM en comparación mujeres sanas que también participaron en el trabajo. Específicamente se detectaron hiperperfusión (exceso de flujo sanguíneo) en la corteza parietal y los surcos precentral y postcentral; todas ellas zonas relacionadas con el dolor y baja circulación sanguínea (o hipoperfusión) en la parte anterior de la corteza temporal izquierda, engranaje fundamental en el mecanismo de control de las emociones. Dichas anormalidades en el flujo cerebral de estos pacientes no dependen de la ansiedad y la depresión y se relacionan con la severidad clínica de la enfermedad (Guedj, et al., 2008).

\section{Causas Biomecánicas del dolor en la FM}

La causa biomecánica que resalta en la FM es el atrapamiento fascial. La fascia es poco conocida en comparación con otras estructuras y es definida como una serie ininterrumpida de tejido conjuntivo fibroso (Rubio y Paredes, 2004; Pilat 2003) de origen embrionario mesodérmico (Smith-Agreda y Ferres-Torres, 2004), formando por capas en dirección oblicua, transversal o circular dándole el aspecto general de espiral (Paoletti, 2004). Su microestructura coloide (Little, 1969) posee propiedades derivadas de la mecanorregulación dada por las características de la tensegridad (Ingber, 2008), donde existe una red de células musculares lisas propias y receptores de Golgi (solamente un 10\% de estos se encuentra en tendones, el 90\% está en la porción muscular de la unión miotendinosa, en cápsulas articulares, ligamentos y fascia), corpúsculos de Paccini (atribuyendo sensibilidad a la vibración), órganos de Ruffini (siendo capaz de responder a impulsos lentos y presiones sostenidas) y terminaciones nerviosas libres de fibras sensitivas tipo III y tipo IV así como receptores del dolor (Pilat 2003; Latash 1998). También existen mecanorreceptores de bajo umbral que responden a un estímulo mecánico extremadamente suave, como la fuerza de una pincelada que puede generar una respuesta autónoma que causa cambios en los ritmos cardíaco y respiratorio así como en la presión arterial (Rubio y Paredes, 2004).

El sistema fascial tiene función de protección (mantiene la integridad anatómica, conservar la forma, amortigua y dispersa impactos), formación de compartimentos corporales (es un elemento "elástico" que cubre todas las estructuras del cuerpo y por tanto es el soporte del equilibrio postural), revestimiento (es una red continua que conecta todos los elementos del cuerpo, uniendo los grupos funcionales con otros anatómicamente muy separados entre sí) y función de coordinación hemodinámica (la fascia proporciona elasticidad y trabaja como una bomba auxiliar que colabora en el envío sangre y linfa desde la periferia hacia el corazón y los ganglios linfáticos respectivamente) (Pilat, 2003).

Para que exista un apropiado intercambio de líquidos corporales debe haber una correcta movilidad fascial que no altere la microcirculación y por tanto el equilibrio de Starling, de lo contrario, se 
puede originar un conjunto de reacciones con progresivo endurecimiento de la sustancia fundamental y acumulación de toxinas al tiempo que no se garantiza una adecuada nutrición de los tejidos (Rubio y Paredes, 2004). Este fenómeno se conoce desde hace mucho tiempo como tixotropía (se hace más fluido cuando se moviliza y se hace más sólido cuando está sin ser afectado) siendo este estado propio de los coloides, característica del tejido fascial (Little, 1969).

Las restricciones del sistema miofascial facilitan la creación de puntos gatillo y producen isquemia, lo que lleva al deterioro en la calidad de la fibra muscular y producción excesiva de colágeno denominada fibrosis, que da lugar a la formación de áreas de atrapamiento y compromiso vascular de las estructuras contráctiles de la zona, es decir, una constante contracción isquémica dolorosa debido a la estimulación de receptores vaniloides. También las terminaciones nerviosas libres son atrapadas causando hipersensibilidad local; conllevando a un fenómeno de sensibilización central que facilita las reacciones referidas al segmento espinal, dando como respuesta una hipertonía en los músculos paravertebrales en el mismo nivel; conduciendo un nuevo ciclo de entrecruzamientos patológicos en las fibras de colágeno de los músculos axiales. El estímulo patológico llega hasta el SNC (estimulando los centros corticales, tálamo y sistema límbico) alterando la calidad de la percepción y las emociones interfiriendo con el proceso total de la homeostasis corporal. La formación de varios puntos de atrapamiento provoca daño celular, liberación de ATP y manifestación dolorosa por estimulación de receptores purinérgicos (Rubio y Paredes, 2004).

Llama la atención en la clínica de la patología, la distribución de los puntos de dolor. Esto se explica cuando un segmento corporal deja de recibir un estímulo adecuado logra patrones de atrapamiento fascial, con la subsecuente alteración en el movimiento. Estos patrones de atrapamiento pueden ser atrapamientos superficiales que se encuentran cerca de las superficies óseas, en inserciones musculares y se forman durante el proceso de transmisión de impulsos mecánicos compensadores y los atrapamientos profundos que implican entrecruzamientos fasciales de grandes masas musculares; favoreciendo la acumulación adiposa, alterando las propiedades del tejido conectivo y perpetuando la disfunción (denominados "puntos de hipersensiblidad" o "tender points"), los cuales coinciden con los puntos recomendados para la exploración (Rubio y Paredes, 2004).

Es imposible explicar un compromiso del sistema fascial basándose únicamente en el aspecto estructural sino también es necesario contemplar el aspecto funcional dado por el componente neurofisiológico. El proceso de adaptación causada por un traumatismo (físico o emocional) cambia la forma del funcionamiento muscular. Normalmente para realizar un movimiento, se utilizan grupos músculos específicos preestablecidos para cada persona y condición (el sujeto posee una forma característica de caminar y podemos identificarla a gran distancia por sus movimientos). Como consecuencia de los atrapamientos fasciales, se acelera el proceso de formación de entrecruzamientos patológicos entre moléculas de colágeno provocando restricción en el deslizamiento de las mismas y reduciendo la amplitud del movimiento en otras zonas de forma que se alteran los patrones de movimiento. Así los movimientos de otras zonas serán también menos efectivos, menos precisos, con mayor gasto energé- tico y supondrán sobrecarga progresiva en diferentes segmentos del sistema musculoesquelético (Rubio \& Paredes, 2004; Pilat, 2003).

Desde un punto de vista biomecánico, un mismo paciente con varios puntos de atrapamiento fascial, tiene varios focos donde se genera tensión las cuales pueden cruzarse y físicamente, estas bandas de tensión actúan como vectores fuerza. Cuando en un mismo campo se manifiestan varios vectores fuerza, los puntos de aplicación en los que coinciden dos o más de ellos sufren una tracción mantenida en varias direcciones lo cual puede dañar el tejido si se supera su límite elástico. Hay que tener en cuenta que los paquetes de fibras se orientan paralelos a la línea de acción de las fuerzas mecánicas lo que les permite trabajar correctamente a tracción, pero las hace muy vulnerables a fuerzas que no sean paralelas a su orientación (Pilat, 2003). También se ha comprobado que los pacientes con FM experimentan desequilibrio de los músculos esqueléticos oculares (causando náuseas o confusión visual al conducir, leer o seguir objetos) al igual que los músculos lisos del ojo ocasionando problemas de foco. Adicional a ello, algunos pacientes sufren de "hipotensión postural de origen neurológico" evidenciándose al ponerse de pie, desencadenando una caída súbita de la tensión arterial y frecuencia cardíaca.

Según Janda, por criterios histológicos y funcionales, los músculos pueden ser tónicos (hiperactivos) y fásicos (inhibidos); los músculos posturales o tónicos responden al prolongado estrés mecánico con tensión y progresiva retracción, mientras que los músculos fásicos responden con un constante debilitamiento creando compensaciones funcionales y por tanto una alteración postural (Rubio y Paredes, 2004; Pilat ,2003). El mecanismo de disfunción fascial con la alteración postural donde el trabajo muscular sufre una alteración en la biomecánica instaurando una zona de atrapamiento fascial, es por ello que los músculos sufren un proceso de estrés mecánico debido a las restricciones de la miofascia. Estos músculos responden al estrés según se lo permitan sus condiciones histológicas (músculos posturales o fásicos) bien con retracción, bien con debilitamiento. Estas respuestas tónicas o fásicas establecen la nueva "postura" del individuo (Rubio y Paredes, 2004).

\section{Principales Alteraciones en la FM}

Alteración en Neurotransmisores del Dolor. La serotonina es deficitaria en un gran número de pacientes. Aunque fue el primer neurotransmisor que se encontró alterado en la FM, posteriormente se halló alteraciones en dopamina, endorfinas y sustancia P (los niveles sistémicos y en el líquido cefalorraquídeo de esta se hallan aumentados de manera importante en comparación con individuos normales) (Tovar, 2005).

Compromisos Neurológicos. Estimaciones por edad y sexo muestran que en la FM vs controles, hay anormalidades neurológicas en múltiples categorías (27 de 29), incluyendo disfunción en nervios craneales en su mayoría en IX y X ( $42 \%$ vs $8 \%$ ), disfunciones sensitivas ( $65 \%$ vs $25 \%$ ) y motoras ( $33 \%$ vs $3 \%$ ), compromiso en la marcha ( $28 \%$ vs $7 \%$ ), fotofobia ( $70 \%$ vs $6 \%$ ), falta de equilibrio ( $63 \%$ vs $4 \%$ ), debilidad muscular ( $58 \%$ vs $2 \%$ ) y sensación de hormigueo en las extremidades ( $54 \%$ vs $4 \%$ ) así como otras menos frecuentes como 
entumecimiento, balance o coordinación deficiente, correlacionados en una evaluación neurológica apropiada (Watson, Buchwald, Goldberg, Noonan y Ellenbogen, 2009).

Alteraciones Musculares. Los pacientes tienen dificultades para mantener un ejercicio intenso con exacerbación posterior del dolor y suelen estar aeróbicamente desacondicionados; también presentan disminución de la fuerza, resistencia y relajación muscular. Se encontró que la fuerza de agarre se reduce en un 40\% $(\mathrm{P}<0,01)$, la fuerza máxima contráctil voluntaria de la mano disminuye significativamente en un $26 \%(\mathrm{P}<0,05)$, la resistencia estática mostro una disminución de 63\% ( $\mathrm{P}<0,004)$ en comparación con controles; la fuerza máxima isométrica e isocinética de extensión de la rodilla es significativamente menor en un $58-66 \%$ y el $41-51 \%$ respectivamente, en comparación con un grupo de controles sanos $(\mathrm{P}<0,01)$ y las biopsias musculares han mostrado hallazgos habituales en personas sedentarias y con falta de entrenamiento muscular (Olsen y Park, 1998). Según lo reportado por Gronemann y colaboradores en 2004, se especula una posible disminución de la oxigenación, cambios en la microestructura muscular consistentes en la fragmentación del ADN y cambios en el tamaño y numero mitocondrial (Sprott, et al., 2004) y con el contenido de colágeno lo que puede favorecer la predisposición a lesiones musculares (Gronemann, Ribel-Madsen, Bartels, Danneskiold-Samsoe y Bliddal, 2004)

También las anomalías metabólicas musculares han mostrado que los niveles de PCr y ATP fueron significativamente más bajos en las muestras de trapecio de los pacientes. La reserva de energía o del denominado potencial de fosforilación (PP) y la capacidad oxidativa total (Vmax) es significativamente menor en pacientes con FM reflejando un deterioro de la fosforilación oxidativa y la síntesis de ATP en los músculos lo que se traduce en fatiga. En general, el mal estado bioenergético de los músculos puede deberse a los niveles bajos de ATP y PCr, la reducción de las reservas de energía (PP) y la capacidad oxidativa (Vmax), todo lo cual podría resultar de la fosforilación oxidativa mitocondrial alterada (Olsen y Park, 1998).

Disautonomía. El compromiso del sistema nervioso simpático se manifiesta por anomalías de la microcirculación cutánea (vasoconstricción) que podrían explicar, la sensibilidad de los puntos característicos de la FM. Mediante la prueba de la mesa basculante a menudo se detecta una hipotensión ortostática asociada a una hiperactividad simpática cardíaca que persiste durante el sueño pero que, paradójicamente, disminuye con el estrés (Thomas, Missounga y Blotman, 2006). Se demostró que la disfunción autonómica por medio de estudios circadianos de la variabilidad del ritmo cardíaco y/o prueba de la mesa basculante puede ser una explicación para los síntomas similares al lupus presentes en algunos pacientes (Martínez-Lavín, Leon, Hermosillo, Pineda y Amigo,1999).

Alteraciones Neuroendocrinas. Se han encontrado alteraciones hormonales, como mala respuesta de las glándulas suprarrenales para la liberación de corticoides, déficit de liberación durante el sueño de somatomedina C (mediadora de la hormona del crecimiento), lo que podría limitar la capacidad de esta última, para reparar los microtraumatismos musculares fisiológicos. Ante diferentes tipos de stress los pacientes no liberarían suficiente cantidad de cortiso- na e igualmente tendrían disminuidos los niveles de hormona de crecimiento (Rubio y Paredes, 2004). Se reconoce que los receptores periféricos sensibles al óxido nítrico de los canales iónicos (ASIC1a) crean microambiente ácido necesario para el desarrollo y mantenimiento de la sensibilización central. Ello también demostró la diferencia in-vivo del músculo con niveles significativamente elevados de sustancia $\mathrm{P}$, gen de la calcitonina péptido relacionado (CGRP), bradiquinina, factor de necrosis tumoral- $\alpha$ (TNF- $\alpha$ ), interleucina-1 $\beta$ (IL-1 $\beta$ ), serotonina y norepinefrina en la región cercana de puntos gatillo miofasciales activos en el músculo trapecio superior (Light, White, Tadler, Iacob y Light, 2012; Podolecki, Podolecki, y Hrycek, 2009; Sumpton y Moulin, 2008).

Alteraciones del Ritmo del Sueño: Los pacientes no llegan a los estadios profundos del sueño (Fase IV), siendo esta la fase "reparadora" del descanso, al observar intrusiones de ondas a en momentos en que no deberían estar presentes. Es decir, en estos pacientes no se produce la desaparición de las ondas a del electroencefalograma (presentes durante la vigilia) durante la fase IV del sueño no REM, cuando deberían de predominar las ondas delta que son más lentas. Esta anomalía se conoce como "sueño alfa-delta", y no es un fenómeno específico de la FM, sino que puede verse también en la artritis reumatoide, personas con estrés emocional por accidentes automovilísticos o laborales, enfermedades febriles, y síndromes post-virales, como el síndrome de fatiga crónica (Millea y Holloway, 2000).

Estos pacientes han mostrado disminución en la variabilidad de la frecuencia cardíaca medida en un periodo de 24 horas, debido a un predominio nocturno de las oscilaciones de la banda de baja frecuencia en consonancia con una modulación simpática exagerada del nodo sinusal. Esta cronobiología anormal podría explicar las alteraciones del sueño y la fatiga y su relación con insuficiencia cardíaca autonómica, mayor riesgo de eventos cardiovasculares y la mortalidad (Da Cunha Ribeiro, et al., 2011). La asociación entre la falta de sueño no-REM y los síntomas podría estar relacionada con una anormalidad en la trasmisión de serotonina, pues se ha demostrado que el p-clorofenilalanina (PCPA) un inhibidor central de la síntesis de serotonina, puede inducir síntomas similares a la FM. Los estudios de neuroimágen han mostrado que estos pacientes tienen un cambio en el flujo sanguíneo cerebral regional relacionada con el dolor de ciertas estructuras, incluyendo los núcleos del tálamo; también presentan cambios de excitabilidad motora de la corteza en los sistemas de excitación/inhibición que son similares a los cambios encontrados en los pacientes con artritis reumatoide (Rubio y Paredes, 2004). El análisis espectral de la variabilidad del ritmo cardíaco puede ser una prueba útil para identificar a los pacientes con FM que tienen disautonomía (Martinez-Lavin, Hermosillo, Rosas y Soto, 1998).

Alteraciones Psicológicas. Estos pacientes presentan un alto nivel de ansiedad y depresión, lo cual es habitual en cualquier persona con dolor crónico. Por otra parte, los factores psicológicos no son causas necesarias ni suficientes de la enfermedad. En un grupo de pacientes ambulatorios, se encontró que solo el 31\% tenían una "alteración psicológica", un 33\% tenía un perfil psicológico normal y un $36 \%$ presentaba una alteración típicamente vista en todos los 
enfermos que presentan dolor crónico, como cáncer o lumbalgias (Thomas, Missounga y Blotman, 2006).

Alteraciones de Piel. La realización de biopsias de piel en estos pacientes ha demostrado que el tejido conectivo de la piel está afectado y que el mastocito es una célula clave en la patogénesis. Son células que actúan en la modulación de procesos inflamatorios, son abundantes en piel y mucosas, tendones, SNC (tálamo, hipotálamo, hipófisis, meninges) y periférico (nervios), además de ganglios. En todas estas zonas, los mastocitos y nervios forman circuitos neuro-inmuno-endocrinos que son fácilmente activados por múltiples factores liberando productos que provocan efectos/estímulos capaces de generar síntomas y la hipersensibilidad típicos de la enfermedad (Rubio y Paredes, 2004). Hay patologías como dermatitis, colon irritable, cistitis intersticial, endometriosis y rinitis entre otros, que se caracterizan por el incremento del número y actividad de mastocitos. Por todo ello la FM podría ser la consecuencia de un incremento del número estos en el tejido conectivo, en respuesta a diversos estímulos (Branco, et al., 2010).

\section{Importancia de la Fisioterapia en la FM}

La Fisioterapia tiene un papel relevante en el manejo del paciente con FM. El fisioterapeuta en su quehacer profesional emplea modalidades físicas de tratamiento como la termoterapia (Gauffin, Hankama, Hannonen, Kautiainen, Pohjolainen y Haanpää, 2013); la crioterapia que logra mejoría en la clínica dolorosa durante unos 90 minutos aproximadamente (Metzger, Zwingmann, Protz y Jackel, 2000; Offenbacher y Stucki, 2000); el masaje que mejora la circulación local muscular y moviliza tejidos afectados (Castro-Sánchez, et al., 2011a; Castro-Sánchez, et al., 2011b; Castro-Sánchez, et al., 2011c; Kalichman, 2010); la electroterapia (Carbonario, Matsutani, Yuan y Marques, 2013) donde se encuentran la electroacupuntura aplicada sobre los puntos dolorosos (sin embargo no se ha valorado su efectividad a largo plazo) (White, 1995; Deluze, Bosia, Zirbs, Chantraine y Vischer, 1992); la Transcutaneous Electrical Nerve Stimulation (TENS) aumenta la microcirculación en las áreas afectadas y promueve la liberación de opioides endógenos en un 70\% de los pacientes (Offenbacher, 2000; Kaada, 1989); los baños hidrogalvánicos que logran mejoría en la sintomatología dolorosa, psicológica y en el sueño a corto plazo (Gunther, Mur, Kinigadner y Miller, 1994). En otras modalidades no existen diferencias significativas en la utilización como los campos magnéticos de polaridad constante y variable, pues ambos disminuyen la clínica de estos pacientes (Alfano, et al., 2001). Por su parte la Fototerapia no se considera una medida eficaz en el tratamiento de esta patología (Pearl, et al., 1996).

Dentro de las modalidades del ejercicio más utilizadas en Fisioterapia, se encuentra el fortalecimiento muscular (Altan, Korkmaz, Bingol y Gunay, 2009; Busch, et al., 2011; Busch, Barber, Overend, Peloso y Schachter, 2007; Cárdenas y Ruiz, 2014; Dupree, Adams, Winters-Stone y Burckhardt, 2006; Häkkinen, Häkkinen, Hannonen y Alen, 2001; Jones, et al., 2008; Jones, Burckhardt, Clark, Bennett y Potempa, 2002; Karper, Jannes y Hampton, 2006; Kayo, Peccin, Sanches y Trevisani, 2012); los ejercicios aeróbicos que mejorar el bienestar y en algunos casos inducen disminución del dolor (Häkkinen, Häkkinen, Hannonen y Alen, 2001; Offenbacher y Stuc- ki, 200o; Mannerkorpi, Nyberg, Ahlmen y Ekdahl, 200o; Ramsay, et al., 2000); la hidroterapia y balneoterapia que han mostrado efectos positivos en la mejoría de la clínica dolorosa (Evcik, Kizilay y Gokcen, 2002). Los baños medicinales e infusiones (incluyendo valeriana y/o aceite de pino) mejoran el bienestar, el sueño y disminuyen la sintomatología (Neumann, et al., 2001; Ammer y Melnizky, 1999).

La educación del paciente con FM (Ang, et al., 2011; Cedraschi, et al., 2004) hace parte de las intervenciones conductuales costo efectivas más aplicadas, la terapia cognitivo-conductual es el método más documentado de la literatura revisada. Generalmente se interviene a tres niveles: el primero son técnicas educativas de conexión cuerpo-mente, la segunda técnicas de relajación, y la tercera movimientos y ejercicios del tipo qigong; el Biofeed-back también ha mostrado su eficacia en el control sintomático, tanto de forma aislada como asociado a ejercicios y técnicas de relajación (Buckelew, et al., 1998). En el abordaje psicoterapéutico, cabe tener en cuenta la predisposición al cambio, como base de la actitud hacia su enfermedad y la disposición a implicarse en su tratamiento pues los resultados no serían los mismos en función del estadio en el que se encuentre el paciente (Dijkstra, Vlaeyen, Rijnen y Nielson, 2001). Otros estudios de menor solidez mencionan la hipnoterapia como un posible tratamiento (Castel, Cascón, Padrol, Sala y Rull, 2012; Derbyshire, Whalley y Oakley, 2009) sin embargo esta debe ser estudiada con mayor rigurosidad.

\section{DISCUSION}

Un aspecto a tener en cuenta es la integración que tiene las causas biomecánicas con las neurofisiológicas en la generación de la FM. Debido a la sensibilización del SNC y periférico, el dolor continuo en los pacientes con FM provoca cambios de plasticidad que puedan sostener el dolor y por lo tanto, mantener un ciclo continuo que es responsable de la cronicidad y condición refractaria de la enfermedad. Por lo tanto, es importante para determinar el grado de los cambios del SNC en estos pacientes, en cuanto a las intervenciones de Fisioterapia. En este contexto, la evaluación y diagnóstico de la sensibilización central y periférica es muy importante porque las neuronas de la médula espinal que normalmente sólo se activan por estímulos nocivos son ahora activadas por estímulos que normalmente no lo son, un fenómeno conocido como alodinia, que en muchos casos requiere una intervención multidisciplinaria. El mismo razonamiento se puede aplicar a la falta de sueño, trastornos psicológicos y otros factores desencadenantes de la recurrencia de los síntomas.

Una comprensión holística de la FM requiere del fisioterapeuta, una evaluación integral del procesamiento neuromuscular y biomecánico, la funcionalidad y el contexto psicosocial para logar reconocerla como una condición compleja y heterogénea donde hay procesamiento anormal del dolor y compromiso del sistema fascial, así como otras características secundarias que alteran la calidad de vida en estos pacientes (Carville, et al., 2007). Las intervenciones no farmacológicas realizado por el fisioterapeuta en dicha patología, se caracterizan por tener beneficios para estos pacientes, disminuyendo el dolor crónico, la fatiga, el desacondicionamiento físico, la inactividad e incluso la kinesiofobia que se acompaña de discapacidad y trastornos psicológicos (Maquet, Demoulin, Croisier y Crielaard, 2007). 
En general, el tratamiento fisioterapéutico puede ser localizado (Electroacupuntura, crioterapia, TENS, termoterapia, terapia manual en puntos gatillo y masoterapia con estiramientos) y tratamiento globalizado (balneoterapia, el masaje relajante, hidroterapia, cinesiterapia, higiene postural y la relajación en agua) y tratamiento fascial (movilización del sistema fascial mediante terapia manual y masaje) (Fernández, 2004; Busch, 2001; Offenbacher, 2000). Se debe complementar toda intervención con estrategias que favorezcan la adherencia al tratamiento propuesto (Arnold, 2006), con el fin de evitar las pérdidas y deserciones. Estas estrategias deben ser de tipo educomunicativas que incluyan material escrito y educación presencial, facilitando la comprensión de la patología, los métodos de tratamiento y actuando como retroalimentación para el refuerzo muscular en el hogar mediante los planes de ejercicio en casa, dando un enfoque múltiple a la intervención (Sañudo, Galiano, Carrasco y de Hoyo, 2010).

Según la evidencia revisada, se sugiere la implementación de estrategias de intervención para estos pacientes, pues esta población reporta que necesitan mayor apoyo del personal de salud (52,3\%), así como profesionales con mayores estudios en el área (50\%), y saber que ésta enfermedad sí existe (30\%) y no es producto de diagnósticos erróneos. Por otro lado, aspectos como tener mejores medicamentos $(15,2 \%)$, más financiación en investigación (13,6\%) o mejor herramientas de diagnóstico $(7,6 \%)$ tiene una puntuación más baja o sea no son tan prioritarios para estos pacientes (Caballero, 2010). A futuro, el manejo de la FM por parte del fisioterapeuta, exigirá una comprensión a profundidad de los mecanismos involucrados en la perpetuación del estímulo doloroso, para realizar intervenciones efectivas y basadas en la evidencia, que mejoren la funcionalidad y calidad de vida en dicha población.

Las investigaciones han demostrado la existencia de anormalidades neuroendocrinas, bioquímicas, musculares y psicológicas asociadas a la FM. Estas anomalías permiten entender las variadas alteraciones que presentan los pacientes y establecer un tratamiento adecuado, donde el fisioterapeuta es pieza clave en el mismo como estudioso del MCH, avalado por la Ley 528 de 1999. La eficacia del tratamiento se encuentra en un enfoque multidisciplinario, uso de fármacos e intervención psicológica que combina terapia cognitivo-conductual acompañada de diversas técnicas de relajación.

La FM es una patología crónica que provoca dolor muscular general, que puede convertirse en discapacitante, comprometiendo la calidad de vida de esta población. Los síntomas más frecuentes son el dolor, la fatiga, trastornos de ánimo y alteraciones del sueño, que en muchos casos son diagnosticados tardíamente o tratados de manera inadecuada. Por esto el fisioterapeuta debe realizar un diagnóstico basado en la objetivación del dolor al presionar entre 11 a 18 puntos predeterminados en estructuras musculotendinosas con dolor bilateral, evaluaciones de los nervios sensitivos y motores así como otras condiciones que limiten el movimiento y la funcionalidad.

No existe un tratamiento definitivo para la FM y las medidas de intervención van dirigidas a disminuir los síntomas, mejorar la calidad de vida, optimización de la funcionalidad, aumentar la capacidad de afrontar la enfermedad y mejorar el bienestar psicológico. Las terapias son poco eficaces de forma aislada y su aplicación conjunta y coordinada constituye una mejor oportunidad para estos pacientes. Sin embargo, no todos tienen la misma evolución ni presentan la misma complejidad. No obstante, el mejor tratamiento que podemos ofrecer a los pacientes con FM, suele ser la comprensión de la enfermedad, ya que responderán automáticamente de forma positiva cuando perciban interés por su mejoría. Esa empatía necesaria para ejercer con profesionalidad la labor del fisioterapeuta, es más económica que los modernos tratamientos farmacológicos y en muchos casos, algo olvidada.

\section{REFERENCIAS}

Asociación Colombiana para el Estudio del Dolor ACED (2010). Quinto estudio Nacional del dolor. Recuperado 29 Agosto de 2012, de: http://www.dolor.org.co/Archivos_Aced/5_Estudio_del_dolor-Osteomuscular.pdf

Alegre, C., García, J., Tomas, M.L., Gómez, J.M., Blanco, E., Gobbo, M., et al. (2010). Documento de Consenso interdisciplinar para el tratamiento de la Fibromialgia. Acta Española de Psiquiatría, $38(2), 108-120$.

Alfano, A.P., Taylor, A.G., Foresman, P.A., Dunkl, P.R, McConnell, G.G., Conaway, M.R., et al. (2001). Static magnetic fields for treatment of fibromyalgia: a randomized controlled trial. Journal Alternative and Complement Medicine, 7(1), 53-64.

Altan, L., Korkmaz, N., Bingol, U. \& Gunay, B. (2009). Effect of Pilates training on people with fibromyalgia syndrome: a pilot study. Archives of Physical Medicine and Rehabilitation, 90, 1983-1988.
Ammer, K., \& Melnizky, P. (1999). Medicinal baths for treatment of generalized Fibromyalgia. Forsch Komplementarmed, 6(2), 80-85.

Ang, D.C., Kaleth, A.S., Bigatti, S., Mazzuca, S., Saha, C., Hilligoss, J., et al. (2011). Research to encourage exercise for Fibromyalgia (REEF): Use of motivational interviewing design and method. Contemporary Clinical Trials, 32(1), 59-68.

Aparicio, V.A., Ortega, F.B., Carbonell-Baeza, A., Femia, P., Tercedor, P., Ruiz, J.R., et al. (2012). Are there gender differences in quality of life and symptomatology between fibromyalgia patients? American Journal of Men's Health, 6(4), 314-319.

Arias, M. (2008). ¿Es la fibromialgia una enfermedad neurológica? Neurología, 23(9), 593-560.

Arnold, L.M. (2006). Biology and therapy of fibromyalgia New therapies in fibromyalgia. Arthritis Research Therapy, 8, 212-232. 
Branco, J.C., Bannwarth, B., Failde, I., Abello, J., Blotman, F., Spaeth, M., et al. (2010). Prevalence of fibromyalgia: a survey in five European countries. Seminars in Arthritis and Rheumatism, 39(6), 448-453.

Buckelew, S.P., Conway, R., Parker, J., Deuser, W.E., Read, J., Witty, T.E., et al. (1998). Biofeedback/relaxation training and exercise interventions for fibromyalgia: a prospective trial. Arthritis Care \& Research, 11(3), 196-209.

Busch, A.J., Webber, S.C., Brachaniec, M., Bidonde, J., Bello-Haas, V.D., Danyliw, A.D., et al. (2011). Exercise therapy for fibromyalgia. Current Pain and Headache Reports, 15(5), 358-367.

Busch, A.J., Barber, K.A., Overend, T.J., Peloso, P.M., \& Schachter, C.L. (2007). Exercise for treating fibromyalgia syndrome. Cochrane Database Systematic Review, 17(4), 1-76.

Caballero, C.V. (2010). Fibromialgia. Conceptos para América Latina. Recuperado 29 Agosto de 2012, de: http://es.scribd.com/Carvicab/ d/33037068-Fibromialgia-Conceptos-actuales-2010\#download.

Cardenas, C. \& Ruiz, J. (2014). Efecto de un programa de actividad física en pacientes con fibromialgia: revisión sistemática. Medicina Clínica (Barcelona), 1-6.

Carbonario, F., Matsutani, L.A., Yuan, S.L. \& Marques, A.P. (2013). Effectiveness of high-frequency transcutaneous electrical nerve stimulation at tender points as adjuvant therapy for patients with fibromyalgia. European Journal of Physical and Rehabilitation Medicine, 49(2), 197-204.

Carville, S.F., Arendt-Nielsen, S., Bliddal, H., Blotman, F., Branco, J.C., Buskila, D., et al. (2007). EULAR evidence based recommendations for the management of fibromyalgia syndrome. Annals of Rheumatic Disease, 1-26.

Castel, A., Cascón, R., Padrol, A., Sala, J. \& Rull, M. (2012). Multicomponent cognitive-behavioral group therapy with hypnosis for the treatment of Fibromyalgia: Long-Term Outcome. Journal of Pain, 13(3), 255-265.

Castro-Sánchez, A,M,, Matarán-Peñarrocha, G.A., Arroyo-Morales, M., Saavedra-Hernández, M., Fernández-Sola, C. \& Moreno-Lorenzo, C. (2011a). Effects of myofascial release techniques on pain, physical function, and postural stability in patients with fibromyalgia: a randomized controlled trial. Clinical Rehabilitation, 25(9), 800-813.

Castro-Sánchez, A.M., Matarán-Peñarrocha, G.A., Sánchez-Labraca, N., Quesada-Rubio, J.M., Granero-Molina, J. \& Moreno-Lorenzo, C. (2011b). A randomized controlled trial investigating the effects of craniosacral therapy on pain and heart rate variability in fibromyalgia patients. Clinical Rehabilitation. 25(1), 25-35.

Castro-Sánchez, A.M., Matarán-Peñarrocha, G.A., Granero-Molina, J., Aguilera-Manrique, G., Quesada-Rubio, J.M. \& Moreno-Lorenzo, C. (2011c). Benefits of massage-myofascial release therapy on pain, anxiety, quality of sleep, depression, and quality of life in patients with fibromyalgia. Evidence Based Complement Alternative Medicine, 2011, 1-9.
Cedraschi, C., Desmeules, J., Rapiti, E., Baumgartner, E., Cohen, P., Finckh, A., et al. (2004). Fibromyalgia: a randomized controlled trial of a treatment programme based on self-management. Annals of Rheumatic Disease, 63, 290-296.

Chaitow, L. (1995). Fibromyalgia and Muscle Pain. Thorsons Pub, 1-205.

Chaves, D. (2013). Actualización en Fibromialgia. ASOCOMEFO, 30(1), 83-88.

Chong, Y.Y. \& Ng, B.Y. (2009). Clinical aspects and management of Fibromyalgia Syndrome. Annals Academy of Medicine Singapore, 38, 967-973.

Congreso de la República de Colombia. Ley 528 Por la cual se reglamenta el ejercicio de la profesión de Fisioterapia, se dictan normas en materia de ética profesional y otras disposiciones. Diario Oficial No. 43711 de 20 de Septiembre de 1999.

Contreras, N.A. \& Tamayo, R.V. (2005). Fibromialgia aspectos clínico-prácticos del diagnóstico y tratamiento. Médica Sur, 12(2), 93-98.

Culpepper, L. (2010). Evaluating the patient with fibromyalgia. Journal Clinical Psychiatry, 71(9), e25.

Da Cunha Ribeiro, R.P., Roschel, H., Artioli, G.G., Dassouki, T., Perandini, L.A., Calich, A.L., et al. (2011). Cardiac autonomic impairment and chronotropic incompetence in fibromyalgia Arthritis Research $\mathcal{E}$ Therapy, 13, 190-195.

Deluze, C., Bosia, L., Zirbs, A., Chantraine, A. \& Vischer, T.L. (1992). Electroacupuncture in fibromyalgia: results of a controlled trial. British Medical Journal, 305(6864), 1249-1252.

Derbyshire, S.W.G., Whalley, M.G. \& Oakley, D.A. (2009). Fibromyalgia pain and its modulation by hypnotic and non-hypnotic suggestion: An fMRI analysis. European Journal of Pain, 13, 542-550.

Dijkstra, A., Vlaeyen, J.W., Rijnen, H. \& Nielson, W. (2001). Readiness to adopt the self-management approach to cope with chronic pain in fibromyalgic patients. Pain, 90(1-2), 37-45.

Dupree, K., Adams, D., Winters-Stone, K. \& Burckhardt, K.S. (2006). A comprehensive review of 46 exercise treatment studies in fibromyalgia (1988-2005). Health and Quality of Life Outcomes, 4, 67-72.

Esteban, B. (2012). Manejar el dolor de manera integral. Publicado 11 de Septiembre de 2012 Vanguardia.com. Recuperado 28 Septiembre de 2012, de: http://www.vanguardia.com/vida-y-estilo/salud/173703-manejar-el-dolor-de-manera-integral.

Evcik, D., Kizilay, B. \& Gokcen, E. (2002). The effects of balneotherapy on fibromyalgia patients. Rheumatology International, 22(2), 56-59.

Fernández de las Peñas, C., Cano Monsalve, G.E., Carravilla Blanco, C., Barrio Lorenzo, L. \& Jerez Martín, M.L. (2004). Opinión de los profesionales sanitarios en relación al tratamiento actual en síndrome fibromiálgico. Fisioterapia y calidad de vida 7(3), 5-12.

REV. COL. REH 2014 || Volumen 13 || Páginas 14 - 25 || 
Forseth, K.O. \& Gran, J.T. (2002). Management of Fibromyalgia what are the Best Treatment Choices? Drugs, 62(4), 577-592.

Gauffin, J., Hankama, T., Hannonen, P., Kautiainen, H., Pohjolainen, T. \& Haanpää, M. (2013). Do fibromyalgia patients use active pain management strategies? A cohort study. Journal of Rehabilitation Medicine, $45(5), 477-480$.

Gronemann, S.T., Ribel-Madsen, S., Bartels, E.M., Danneskiold-Samsoe, B. \& Bliddal, H. (2004). Collagen and muscle pathology in fibromyalgia patients. Rheumatology (Oxford), 43(1), 27-31.

Guedj, E., Cammilleri, S., Niboyet, J., Dupont, P., Vidal, E., Dropinski, J.P., et al. (2008). Clinical correlate of brain SPECT perfusion abnormalities in Fibromyalgia. Journal of Nuclear Medicine. 49(11), 1798-1803.

Gunther, V., Mur, E., Kinigadner, U. \& Miller, C. (1994). Fibromyalgia the effect of relaxation and hydrogalvanic bath therapy on the subjective pain experience. Clinical Rheumatology, 13(4), 573-578.

Häkkinen, A., Häkkinen, K., Hannonen, P. \& Alen, M. (2001). Strength training induced adaptations in neuromuscular function of premenopausal women with fibromyalgia: comparison with healthy women. Annals of Rheumatic Disease, 6o(1), 21-26.

Hernández, J.J. \& Moreno, C. (2008). Fibromialgia en la mujer. Asociación Colombiana para el Estudio del Dolor ACED, 197-214.

Hurtado, Y. (2012) El 80\% de los casos de fibromialgia en mujeres es subdiagnósticado. Periódico electrónico CronicadelQuindio.com. Publicado 13 de Marzo de 2012. Recuperado 19 Octubre de 2012, de: http:// www.cronicadelquindio.com/noticia-completa-titulo-el_80_de_ los_casos_de_fribromialgia_en_mujeres_es_subdiagnosticado-seccion-general-nota-43868.htm

Imamura, M., Cassius, D.A. \& Fregni, F. (2009). Fibromyalgia: From treatment to rehabilitation. European Journal of Pain, 3(2), 117-122.

Ingber, D.E. (2008). Tensegrity and Mechanotransduction. Journal of Bodywork and Movement Therapies, 12(3), 198-200.

Jahan, F., Nanji, K., Qidwai, W. \& Qasim, R. (2012). Fibromyalgia Syndrome: An overview of pathophysiology, diagnosis and management. Oman Medical Journal, 27(3), 192-195.

Jones, K.D., Burckhardt, C.S., Clark, S.R., Bennett, R.M. \& Potempa, K.M. (2002). A randomized controlled trial of muscle strengthening versus flexibility training in fibromyalgia. Journal of Rheumatology, 29(5), 1041-1048.

Jones, K.D., Burckhardt, C.S., Deodhar, A.A., Perrin, N.A., Hanson, G.C. \& Bennett, R.M. (2008). A six-month randomized controlled trial of exercise and pyridostigmine in the treatment of fibromyalgia. Arthritis \& Rheumatism, 58, 612-622.
Kaada, B. (1989). Treatment of fibromyalgia by low-frequency transcutaneous nerve stimulation. Tidsskr Nor Laegeforen, 109(29), 2992-2995.

Kalichman, L. (2010). Massage therapy for fibromyalgia symptoms. Rheumatology International, 30(9), 1151-1157.

Karper, W.B., Jannes, C.R.\& Hampton, J.L. (2006). Fibromyalgia syndrome: the beneficial effects of exercise. Rehabilitation Nursing, 31(5), 193-198.

Kayo, A.H., Peccin, M.S., Sanches, C.M. \& Trevisani, V.F. (2012).Effectiveness of physical activity in reducing pain in patients with fibromyalgia: a blinded randomized clinical trial. Rheumatology International, 32(8), 2285-2292.

Latash, M.L. (1998). Neurophysiological Basis of Movement. Editorial Human Kinetics.

Lee, K. (2006). Historia de la Fibromialgia. Recuperado o3 Mayo de 2012, de: http://chronicfatigue.about.com/od/fibromyalgia/p/historyfm.htm.

Light, K.C., White, A.T., Tadler, S., Iacob, E. \& Light, A.R. (2012). Genetics and Gene Expression Involving Stress and Distress Pathways in Fibromyalgia with and without Comorbid Chronic Fatigue Syndrome. Pain Research and Treatment, 1-13.

Little, K.E. (1969). Toward more effective Manipulative management if chronic myofascial strain and stress syndromes. Journal American Osteopathic Association, 68, 675-685.

Mannerkorpi, K., Nyberg, B., Ahlmen, M. \& Ekdahl, C. (2000). Pool exercise combined with an education program for patients with fibromyalgia syndrome. A prospective, randomized study. The Journal of Rheumatology, 27(10), 2473-2481.

Maquet, D., Demoulin, C., Croisier, J.L. \& Crielaard, J.M. (2007). Benefits of physical training in fibromyalgia and related syndromes. Annales de réadaptation et de médecine physique, 50, 363-368.

Martin, D.P., Sletten, C.D., William, B.A. \& Berger, I.H. (2006). Improvement in Fibromyalgia Symptoms with Acupuncture: Results of a Randomized Controlled Trial. Mayo Clinic Proceedings, 81(6), 749-757.

Martinez-Lavin, M., Leon, A., Hermosillo, A.G., Pineda, C. \& Amigo, M.C. (1999). The dysautonomia of fibromyalgia may simulate lupus. Journal of Clinical Rheumatology, 5, 332-334.

Martinez-Lavin, M., Hermosillo, A.G., Rosas, M. \& Soto, M.E. (1998). Circadian studies of autonomic nervous balance in patients with fibromyalgia. A heart rate variability analysis. Arthritis \& Rheumatology, 41(11), 1966-1971.

Metzger, D., Zwingmann, C., Protz, W. \& Jackel, W.H. (200o). Whole-body cryotherapy in rehabilitation of patients with rheumatoid diseases pilot study. Rehabilitation, 39(2), 93-100. 
Millea, P. \& Holloway, R. (2000). Treating Fibromyalgia. American family physician, 1575-1582.

Moretti, F.A., Ezequiel, R., Marvulle, V., Pollak, D.F. \& Riera, R. (2011). Assessing knowledge on Fibromyalgia among internet users. Revista Brasileira Reumatologia, 51(1), 7-19.

Neumann, L., Sukenik, S., Bolotin, A., Abu-Shakra, M., Amir, M., Flusser, D. \& Buskila, D. (2001). The effect of balneotherapy at the Dead Sea on the quality of life of patients with fibromyalgia syndrome. Clinical Rheumatology, 20(1), 15-19.

Nijs, J., Mannerkorpi, K., Descheemaeker, F. \& Van Houdenhove, B. (2010). Primary care physical therapy in people with fibromyalgia: opportunities and boundaries within a monodisciplinary setting. Physical Therapy. 90(12), 1815-1822.

Offenbacher, M. \& Stucki, G. (2000). Physical therapy in the treatment of fibromyalgia. Scandinavian journal of rheumatology, 113, 78-85.

Olsen, N.J. \& Park, J.H. (1998). Skeletal muscle abnormalities in patients with Fibromyalgia. American Journal of Medical Sciences, 315(6), 351-363.

Paoletti, S. (2004). El papel de los tejidos en la mecánica humana. $3^{\mathrm{a}}$ Edición. Editorial Paidotribo: Barcelona.

Pearl, S.J., Lue, F., MacLean, A.W., Heslegrave, R.J., Reynolds, W.J. \& Moldofsky, H. (1995). The effects of bright light treatment on the symptoms of fibromyalgia. Journal of Rheumatology, 23(5), 896-902.

Perrot, S. (2008). Fibromyalgia syndrome: a relevant recent construction of an ancient condition? Current Opinion Support Palliative Care, $2(2), 122-127$.

Pilat, A. (2003). Terapias Miofasciales: Inducción Miofascial. Editorial McGraw-Hill / Interamericana de España, S.A.

Podolecki, T., Podolecki, A. \& Hrycek, A. (2009). Fibromyalgia: pathogenetic, diagnostic and therapeutic concerns. Pol Arch Med Wewn, 119(3), 157-161.

Ramsay, C., Moreland, J., Ho, M., Joyce, S., Walker, S. \& Pullar, T. (2000). An observer-blinded comparison of supervised and unsupervised aerobic exercise regimens in fibromyalgia. Rheumatology (Oxford), 39(5), 501-505.

Rivera, J., Alegre, C., Nishishinya, M.B. \& Pereda, C.A. (2006). Evidencias terapéuticas en Fibromialgia. Reumatol Clin, 2(1), 34-37.

Rubio, J. \& Paredes, J.A. (2004). Fibromialgia. Propuesta de modelo fisiopatológico fascial. Madrid, España: Editorial U San Pablo CEU.

Sañudo, B., Galiano, D., Carrasco, L. \& de Hoyo, M. (2010). Evidencias para la prescripción de ejercicio físico en pacientes con fibromialgia. Revista Andaluza de Medicina del Deporte, 3(4),159-169.
Schiltenwolf, M., Häuser, W., Felde, E., Flügge, C., Häfner, R., Settan, M., et al. (2008). Physiotherapy, exercise and strength training and physical therapies in the treatment of fibromyalgia síndrome. Schmerz, 22(3), 303-312.

Sluka, K.A. (2012). Mechanism and management of pain for the Physical Therapist. 1th Edition. IASP Press: Seattle.

Smith-Agreda, V. \& Ferres-Torres, E. (2004). Principios de anatomofisiopatología. Editorial Paidotribo: Barcelona.

Smith, H.S., Harris, R. \& Clauw, D. (2011). Fibromyalgia: An Afferent Processing Disorder Leading to a Complex Pain Generalized Syndrome. Pain Physician, 14, 217-245.

Sprott, H., Salemi, S., Gay, R.E., Bradley, L.A., Alarcón, G.S., Oh, S.J., et al. (2004). Increased DNA fragmentation and ultrastructural changes in fibromyalgic muscle fibres. Annals of the Rheumatic Diseases, 63(3), 245-251.

Sumpton, J,E, \& Moulin, D.E. (2008). Fibromyalgia: Presentation and management with a focus on pharmacological treatment. Pain Respiratory Management, 13(6), 477-483.

Thomas, E., Missounga, L. \& Blotman, F. (2006) Fibromialgia. Kinesiterapia y Medicina física, $1-7$.

Tovar, M.A. (2005). Fibromialgia. Colombia Médica, 36(004), 287-291.

Villagrán, J.M., Páez, M., Campo, F., Pérez, J. \& Salaberri, J. (200o). Aspectos psicopatológicos de la Fibromialgia, Archivos de Psiquiatria, 63(2), 159-182.

Watson, N.F., Buchwald, D., Goldberg, J., Noonan, C. \& Ellenbogen, R.G. (2009). Neurologic signs and symptoms in Fibromyalgia. Arthritis $\mathcal{E}$ Rheumatism, 60(9), 2839-2844.

White, A. The fibromyalgia syndrome. (1995). Electroacupuncture is a potentially valuable treatment. BMJ, 310(6991),1406.

Wolf, M. (2009). Historia de la Fibromialgia. Recuperado 27 Septiembre de 2012, de: http://fibromialgico.blogspot.com/2009/03/historia-de-la-fibromialgia.html

Wolfe, F., Clawn, D.J., Fitzcharles, M.A., Goldenberg, D.L., Katz, R.S., Mease, P., et al. (2010). The American College of Rheumatology preliminary diagnostic criteria for Fibromyalgia and measurement of symptom severity. Arthritis Care \& Research, 62, 600-610.

Wolfe, F., Smythe, H.A., Yunus, M.B., Bennett, R.M., Bombardier, C., Goldenberg, D.L., et al. (1990). The American College of Rheumatology 1990 criteria for the classification of fibromyalgia: report of the Multicenter Criteria Committee. Arthritis \& Rheumatology, 33, 160-172.

REV. COL. REH 2014 || Volumen 13 || Páginas 14 - 25 || 